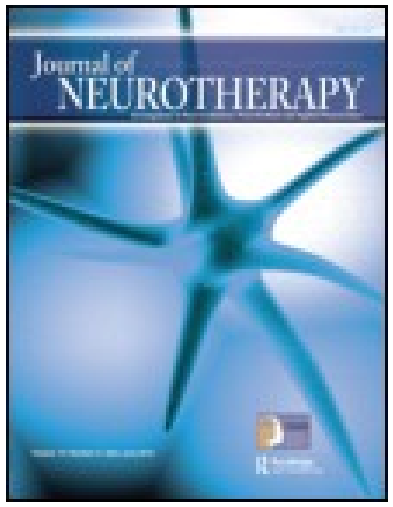

J ournal of Neurotherapy: Investigations in Neuromodulation, Neurofeedback and Applied Neuroscience

\title{
Alpha EEG Activity Depends on the Individual Dominant Rhythm Frequency
}

Olga Mikhailovna Bazanova PhD, DSci a

a Biofeedback Software Laboratory, State Research Institute of Molecular Biology and Biophysics, Siberian Branch, Russian Academy of Medical Sciences, Novosibirsk , Russia Published online: 21 Nov 2012.

To cite this article: Olga Mikhailovna Bazanova PhD, DSci (2012) Alpha EEG Activity Depends on the Individual Dominant Rhythm Frequency, J ournal of Neurotherapy: Investigations in Neuromodulation, Neurofeedback and Applied Neuroscience, 16:4, 270-284, DOI: $10.1080 / 10874208.2012 .730786$

To link to this article: http:// dx.doi.org/ 10.1080/ 10874208.2012.730786

PLEASE SCROLL DOWN FOR ARTICLE

(c) International Society for Neurofeedback and Research (ISNR), all rights reserved. This article (the "Article") may be accessed online from ISNR at no charge. The Article may be viewed online, stored in electronic or physical form, or archived for research, teaching, and private study purposes. The Article may be archived in public libraries or university libraries at the direction of said public library or university library. Any other reproduction of the Article for redistribution, sale, resale, loan, sublicensing, systematic supply, or other distribution, including both physical and electronic reproduction for such purposes, is expressly forbidden. Preparing or reproducing derivative works of this article is expressly forbidden. ISNR makes no representation or warranty as to the accuracy or completeness of any content in the Article. From 1995 to 2013 the J ournal of Neurotherapy was the official publication of ISNR (www. Isnr. org); on April 27, 2016 ISNR acquired the journal from Taylor \& Francis Group, LLC. In 2014, ISNR established its official open-access journal NeuroRegulation (ISSN: 2373-0587; www. neuroregulation. org).

THIS OPEN-ACCESS CONTENT MADE POSSIBLE BY THESE GENEROUS SPONSORS

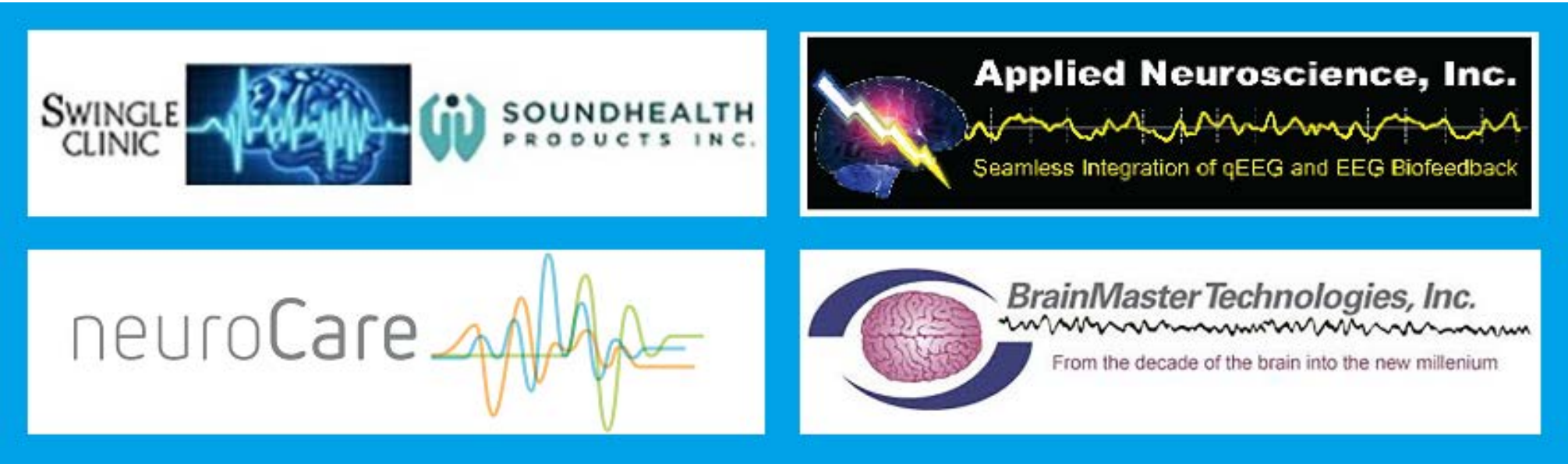




\title{
REVIEW ARTICLE
}

\section{ALPHA EEG ACTIVITY DEPENDS ON THE INDIVIDUAL DOMINANT RHYTHM FREQUENCY}

\author{
Olga Mikhailovna Bazanova \\ Biofeedback Software Laboratory, State Research Institute of Molecular Biology and Biophysics, \\ Siberian Branch, Russian Academy of Medical Sciences, Novosibirsk, Russia
}

Exploring the role of the electroencephalographic (EEG) alpha oscillations in the processes of central and autonomic regulation of the cognitive and psychomotor performance generates considerable interest. However, until now there was no accurate definition of what alpha activity is, and which indices characterize it. This is a theoretical article that attempts to define EEG alpha-activity phenomena; define their physical, molecular, and morphological nature; and highlight the difference in its indices connected with different individual alpha frequencies and their role in optimal functioning. Specifically, this article examines individual alpha activity indices: (a) the individual alpha peak frequency; (b) activation magnitude measured by estimating the amount of alpha amplitude suppression in response to eyes-open and individual alpha band width; and (c) three alpha "autorhythmicity" indices: intraspindle amplitude variability, spindle length, and steepness. Throughout, the article provides a number of suggestions on how alpha activity indices are connected with the individual spectral distribution of the alpha frequency and why these individual alpha activity peculiarities are important for neurofeedback training effectiveness.

\section{INTRODUCTION}

Since the time of the eminent Russian physiologist Ivan Michailovich Sechenov, it was pointed out that the body is an amazing system with many complexities and a special potential for improving itself with feedback connections (Bernstein, 1967). Such self-improvement may target what is now called "peak performance" (R. N. Singer, 2002; Vernon, 2009) or "optimal functioning" (Keefe, 1978) and can be achieved with biofeedback technology (Bazanova, Mernaya, \& Shtark, 2009; Hanslmayr, Sauseng, Doppelmayr, Schabus, \& Klimesch, 2005; Vernon \& Gruzelier, 2008). The recent identification of specific brain signatures involved in optimal cognitive and psychomotor functioning demonstrates its association with general enhancement of the electroencephalographic (EEG) alpha activity (Bazanova \& Aftanas, 2008; Bazanova, Gvozdev, Mursin, Verevkin, \& Shtark, 2003; Hummel et al., 2004; Baumeister, Reinecke, Liesen, \& Weiss, 2008). Thus, this article argues that EEG alpha activity reflects a certain type of self-control (top-down) process and that the properties of this processrelated activity may vary among individuals.

Despite the key role of EEG alpha activity in optimal functioning - known since Berger's time- it is not clear which qualities characterize it (e.g., increasing or decreasing alpha amplitude, left or right shift of spectral alpha

Received 28 August 2012; accepted 6 September 2012.

This research was supported by Russian Humanitarian Science Foundation Grants 10-06-00265a and BIAL Grant 45/08. I thank Professor L. I. Aftanas for discussion and comments on this manuscript. I also thank Dr. Efthymios Angelakis for his help with English language editing.

Address correspondence to Olga Mikhailovna Bazanova, PhD, DSci, Timakova, 2, Institute of Molecular Biology and Biophysics, Siberian Branch, Russian Academy of Medical Science, 630117, Novosibirsk, Russia. E-mail: bazanova_olgamih@mail.ru 
peak frequency, or alpha synchronization or desynchronization). Therefore, this review aims to determine (a) what phenomena could be called "EEG alpha activity" and what brain mechanisms are underlying this, (b) which EEG parameters characterize EEG alpha activity, and (c) their intra- and interindividual variability.

From physics we know that wave activity is characterized by three main parameters: frequency, amplitude, and phase. So it is possible to define three main index groups of the alpha wave activity (Figure 1):

1. Frequency of the dominant posterior EEG rhythm (Niedermeyer, 1999; Nunez, Wingeier, \& Silberstein, 2001), which is assessed as individual alpha peak frequency in an eyes-closed condition (IAPF; Angelakis, Stathopoulou, \& Frymiare, 2007; Bazanova, 2011; Hooper, 2005).

2. Responsiveness/activation, which is assessed by alpha amplitude suppression in response to opening of the eyes (Barry, Clarke, Johnstone, Magee, \& Rushby, 2007; Del Percio et al., 2011; Kirshfieldt, 2005; Nunez et al., 2001), and width of the frequency range of amplitude suppression, or individual alpha bandwidth (Bazanova \& Aftanas, 2006, 2008).

3. "Autorhythmicity" (or bursting or spindle forming process), which is a result of phase resetting (Ivanitsky \& Lebedev, 2007; Thatcher, North, \& Biver, 2008; Timofeev et al., 2002).

\section{THE IAPF}

Many empirical studies present evidence that IAPF varies intraindividually as a function of age-it increases through childhood until pubertal age (Bazanova, 2008; Stroganova, Orekhova, \& Posikera, 1999) and decreases after 40 years of age (Clark et al., 2004). A few studies have shown the hormonal influence on IAPF - it increases with progesterone activity enhancement during menstrual cycle in women (Baker \& Colrain, 2010; Bazanova \& Mernaya, 2008; Creutzfeldt et al., 1976; SolisOrtiz, Guevara, \& Corsi-Cabrera, 2004), and in conditions when cortisol blood level increases (Becker, Creutzfeldt, Schwibbe, \& Wuttke, 1980; Tops, van Peer, Wester, Wijers, \& Korf, 2006). Moreover, IAPF varies depending on

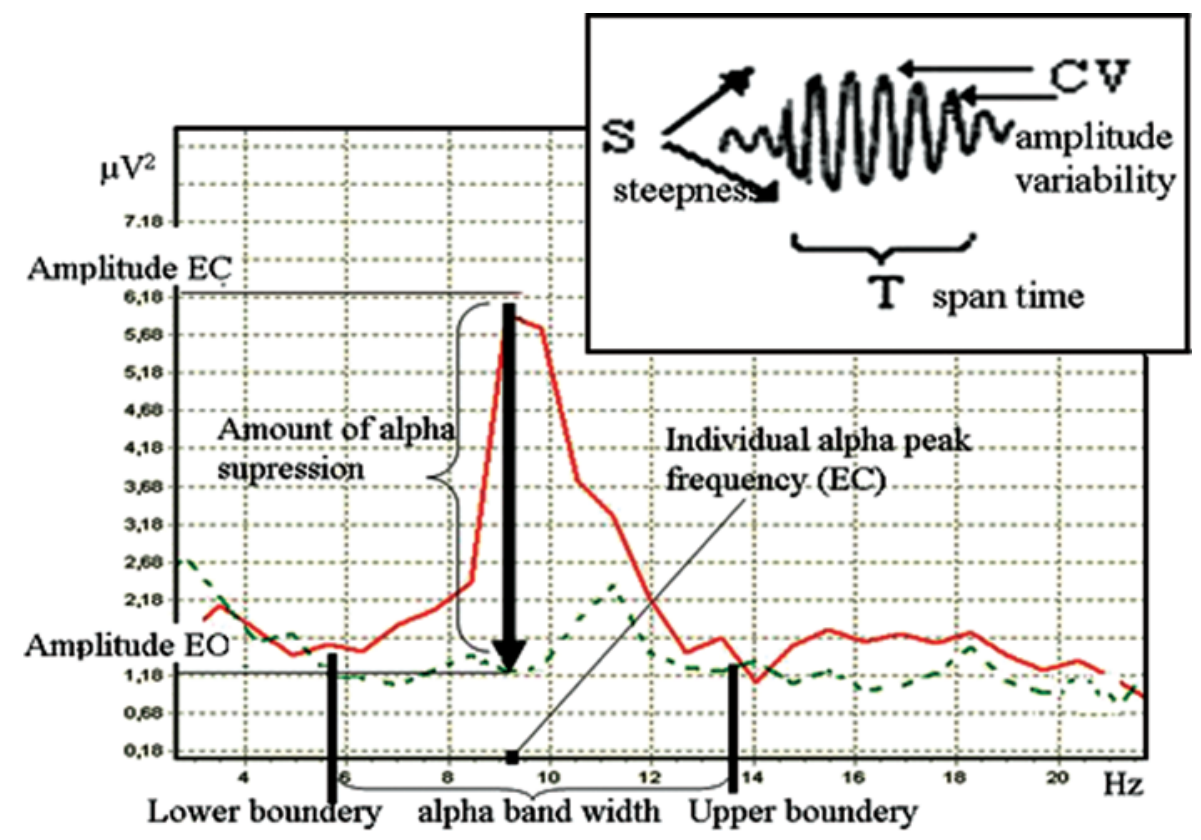

FIGURE 1. Alpha activity indices. Note. EC = eyes closed - bold line spectra; EO=eyes open - dotted line spectra). (Color figure available online.) 
the state individuals are during resting (baseline) conditions, and their personal involvement in a cognitive performance task (Hummel et al., 2004; Klimesch, Sauseng, \& Hanslmayr, 2007; Ng \& Raveendran, 2007). Good task performance is related with higher IAPF (Angelakis, Lubar, \& Stathopoulou, 2004; Klimesch, Schimke, \& Pfurtscheller, 1993), and neurofeedback training (NFT) directed on increasing IAPF may be used to improve cognitive performance (Angelakis et al., 2007). A decrease in IAPF is related to a drop in performance (Klimesch et al., 1993) or to fatigue (Ng \& Raveendran, 2007).

IAPF doesn't always increase after NFT to enhance the upper alpha power. In a recent study investigating the enhancement of the upper alpha power with NFT and its impact on the peak performance of musical achievement, we showed that IAPF increased only in students with a resting baseline alpha frequency lower than $10 \mathrm{~Hz}$ (LAF subjects). On the contrary, students with original IAPF higher than $10 \mathrm{~Hz}$ (HAF) did not change or even decreased their IAPF in a post-NFT resting condition (Bazanova et al., 2009). Hence, it could be proposed that differences in alpha peak frequency reflect different mechanisms of brain activation and alpha wave generation.

In a study with twins, Gavrish and Malykh (1994) proposed that the nature of different alpha-frequency components is heritable. Other genetic studies confirm the high heritability of the resting IAPF (Anohkin, Muller, Lindenberger, Heath, \& Myers, 2006; Smit, Wright, Hansell, Geffen, \& Martin, 2006). It is now evident that IAPF reflects individual genetic influences on the underlying neural mechanisms of the alpha waves generation (Steriade, 1999; Steriade \& Timofeev, 2003).

Evidence from animal models suggests that alpha wave frequency is a result of the local cortical network tuning, which depends on Ca2+ T-channels activity (Lopes da Silva, 1991; Steriade, 1999; Steriade \& Timofeev, 2003). It was shown that blocking of the Ca2+ oscillations (an intracellular signal, rather than an EEG one) is associated with blocking of the spindle wave refractory period, which lies in the origin of the 6 to $10 \mathrm{~Hz}$ oscillations (Lüthi \& McCormick, 1998). So frequency of the Ca2+ oscillations determines the dominant brain frequency (Lüthi \& McCormick, 1998). These experimental results support the hypothesis by Michael Livanov and John Eccles about the inhibitory role of delaying the rhythmic waves generated in the thalamus for brain and mind self-regulation (Eccles, 1994; Livanov, 1984; Livanov \& Dumenko, 1987).

Among different alpha frequency measurements (individual alpha peak frequency, mean and center of gravity), IAPF is preferential for studying between individual differences (Hooper, 2005). As shown by Greg Hooper (2005), the contrasts in upper and lower alpha bands may be explained in terms of the variability or distribution of the peak alpha frequency itself. However, the definition of appropriate frequency bands and the choice of recording reference limit the interpretability of quantitative EEG. The analysis of fixed frequency bands could therefore blur the real alpha peak, masking the age- or function-related differences. To study the endophenotypic qualities of the alpha frequency, we asked, "In which experimental condition and where topographically is IAPF mostly stable and reproducible?" We then analyzed the "test-retest" EEGs from 96 male subjects aged 26 to 40 recorded twice over 14 to 15 days. EEG was recorded in resting eyesclosed and eyes-open conditions and analyzed in a fixed standard $8-12 \mathrm{~Hz}$ frequency band, as well as in an individually determined alpha frequency band. It appeared that the intraindividual correlation coefficient was strongest in posterior brain areas, in the eyes-closed condition, and assessed with individually determined alpha band. The weakest intraindividual correlation coefficient was calculated in anterior areas, in the eyes open condition, and using the fixed standard $8-12 \mathrm{~Hz}$ alpha range (Bazanova, 2011). We did not find the laterality influence on the IAPF mean. Bodenmann and coauthors showed, too, that genotypedependent differences in IAPF were independent from EEG recording location (Bodenmann et al., 2009). So it could be 
concluded that alpha generation in healthy individuals has a universal origin.

What is the functional role of the human alpha rhythm frequency? An eminent Russian psychophysiologist, Artur Lebedev (1994, 2006), has proposed that "cyclical oscillations in the alpha rhythm determine the capacity and speed of working memory. The higher the frequency the greater the capacity and the speed of memory" (Lebedev, 1994, p. 257). Then, Klimesch et al., (1993) argued that thalamo-cortical feedback loops oscillating within the alpha frequency range allow searching and identification of encoded information. He speculated that faster oscillating feedback loops would correspond to faster access to encoded information. These theories are supported by the results of the following studies. Klimesch et al. (1993) found that the alpha peak frequency of good working memory performers lies about $1 \mathrm{~Hz}$ higher than that of poor working memory performers. We had shown that reading is better in musicians with higher, rather than lower, IAPF (Bazanova et al., 2009). The same results were revealed by the functional Val158Met polymorphism of the catechol-Omethyltransferase (COMT) gene, where LAF$\mathrm{Val} / \mathrm{VAL}$ homozygote subjects demonstrated a lower score in a counting task than HAF-Met/ MET homozygote young men (Bodenmann et al., 2009).

With the question "Are smarter brains running faster?" Posthuma, Neale, Boomsma, and de Geus (2001) concluded that both peak frequency and the dimensions of IQ were highly heritable (range $=66-83 \%$ ). But, in this study, a large part of the genetic variance in alpha peak frequency as well as in working memory and processing speed was due to nonadditive factors. Moreover, there was no evidence of a genetic correlation between alpha peak frequency and any of the four WAIS dimensions. So there must be additional EEG indices predicting cognitive ability.

In our experiment using the median of posterior IAPF from a resting eyes-closed condition, 96 healthy male subjects were divided into two groups: those with low (LAF, IAPF $<10 \mathrm{~Hz})$ and those with high (HAF, IAPF

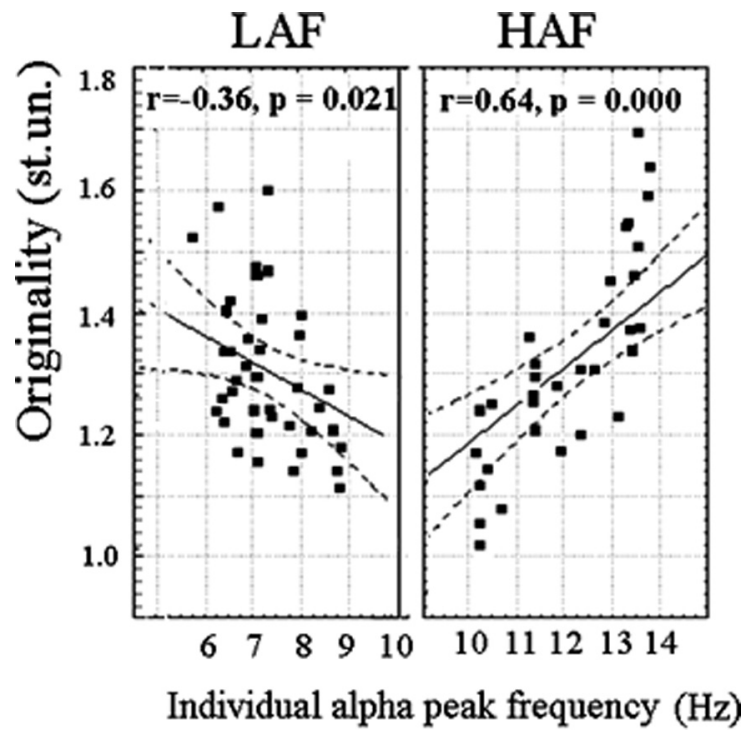

FIGURE 2. The interrelationships between individual alpha peak frequency and originality in a nonverbal task in low (LAF) and high (HAF) alpha frequency male subjects.

$\geq 10 \mathrm{~Hz}$ ) alpha frequency. It appeared that $\mathrm{LAF}$ and HAF subjects differed in psychometric strategies for achieving success in nonverbal creative tasks. LAF subjects emphasized originality, whereas HAF emphasized fluency in reaching the same performance score in the Torrance test (Bazanova \& Aftanas, 2008). However, the subjects with highest and lowest IAPF level showed the highest originality score in a nonverbal creativity task (see Figure 2).

We proposed that different behavior strategies observed in LAF and HAF subjects could be due to (a) genetic factors for individual patterns of spontaneous waking EEG and (b) different neurophysiological mechanisms of brain activation in low and high alpha frequency ranges (Kirshfeldt, 2005).

\section{THE RESPONSIVENESS/ACTIVATION MAGNITUDE}

Vaez Mousavi and colleagues conceptualized two aspects of the energetic state, "arousal" and "activation" (Vaez Mousavi, Barry, Rushby, \& Clarke, 2007). "Arousal" has been defined as the energetic state at any particular time, and task-related "activation" as the task-related change in state from resting baseline to the task 
situation. Accordingly, EEG data activation magnitude indicates the amount of alpha amplitude suppression in response to eyes opening (Barry et al., 2007). Recent investigations showed that EEG measures of visual and cognitive activation are a promising avenue of study in the search for putative endophenotypes for individual cognitive strategy type (Ivanitsky, Ivanitsky, \& Sysoeva, 2009; Loo \& Smalley, 2008) or for ADHD (Loo et al., 2010).

Doppelmayr and colleagues found a significant interaction of task difficulty and activation in the upper alpha band, indicating both a weaker activation for the highly intelligent subjects during the easy tasks and a significant increase from easy to difficult tasks for this group only (Doppelmayr, Klimesch, Hodlmoser, Sauseng, \& Gruber, 2005). Recently, Del Percio and coauthors tested the hypothesis that, compared with nonathletes, elite athletes are characterized by a reduction of reactivity of EEG alpha rhythms (about $8-12 \mathrm{~Hz}$ ) to eyes opening in the condition of resting state, as a possible index of spatially selective cortical activation (i.e., "neural efficiency"; Del Percio et al., 2011). It could be proposed that activation magnitude variability is dependent on cognitive task difficulty and professional experience and is particularly associated with reaction time (Vaez Mousavi et al., 2007) and psychomotor task performance (Bazanova, Kondratenko, Kondratenko, Mernaya, \& Zhimulev, 2007). Moreover, the amount of alpha suppression in response to eyes opening depends on the neurohumoral status (Bazanova \& Mernaya, 2008; Mantanus, Ansseau, Legros, \& Timsit-Berthier, 1988) and even the direction of eye movement (V. Kaiser, Clemens, Leeb, Neuper, \& Pfurtscheller, 2009). A decreased amount of alpha suppression in response to eyes opening was demonstrated in spinal-cord-injured individuals (Thuraisingham, Tran, Boord, \& Craig, 2007) in comparison with healthy participants, and in patients with attention deficit (Barry et al., 2003). Increased activation magnitude is a result of upper alpha stimulating neurofeedback training (Bazanova et al., 2009; Schmelkina, 1999). Of interest, reactivity to eyes opening increases from 3 to 20 years of age and decreases after 40 years of age in HAF but doesn't change in LAF healthy male subjects (Bazanova, 2008).

Based on simulations of the EEG, Pfurtscheller and Lopes da Silva (1999) hypothesized that with an increasing number of interconnecting neurons and therewith an increasing number of coherently activated neurons, the amplitude increases and the frequency decreases. Furthermore, they support that EEG amplitude is negatively correlated with frequency not only between frequency bands, but also within frequency subcomponents (Pfurtscheller \& Lopes da Silva, 1999).

Measuring ERPs from the visual cortex after the presentation of checkerboard stimuli, Mazaheri and Jensen (2006) found that alpha $(M=10 \mathrm{~Hz})$ oscillations preserve their phase relationship after visual stimuli with respect to before the stimuli, while theta $(M=6.6 \mathrm{~Hz})$ phase-resetting oscillations are responsible for visually evoked responses. They concluded that alpha activity plays a modulatory role in perception rather than generating visually evoked responses (Mazaheri \& Jensen, 2006). In EEG-fMRI coupled investigations, Laufs and coauthors (2006) showed that spontaneous reductions in alpha amplitude over occipital areas is connected to fronto-parietal fMRI activation when (a) theta power is low and beta power is high and (b) PAF is high, while it is connected to occipital fMRI activation when beta power is low and theta power is high and PAF is low and more variable. These different interrelations between the amount of alpha amplitude suppression (desynchronization) and other EEG indices in low- and highfrequency ranges is consistent with the idea that alpha desynchronization serves an integrative role through a corticocortical "gating" (Kirshfeldt, 2005; Tenke \& Kayser, 2005). This supports the idea that the neurophysiological mechanisms by which activation is generated are different in LAF and HAF subjects (Bazanova, Jafarova, Mazhirina, Mernaya, \& Shtark, 2008), which is what was theoretically supported in previous investigations (Kirshfeldt, 2005; Mazaheri \& Jensen, 2006). 
Until now, the alpha frequency range boundaries were defined at $8-12 / 13 \mathrm{~Hz}$ on the basis of general agreement, without a theoretical basis and without respect to functional features of alpha rhythm reactivity to visual stimulation (D. A. Kaiser, 2001; Thatcher et al., 2008; Arns, Gunkelman, Breteler, \& Spronk, 2008). Meanwhile, the individual alpha bandwidth (IABW) is dependent on (a) the age - it enlargers from 3 until 20 years (Bazanova, 2008); (b) neurohumoral status in women - in high estrogen and progesterone stages, alpha band is wider than in lower hormonal phases during the menstrual cycle (Bazanova \& Mernaya, 2008); and (c) gender - women have a higher low alpha band boundary frequency than men (Bazanova \& Mernaya, 2008; Carrier, Land, Buysse, Kupfer, \& Monk, 2001). Moreover, individual alpha frequency bandwidth could vary in accordance with brain activation (Bazanova \& Aftanas, 2008; Kaiser, 2001; see Figure 3).

For example, IABW is wider in highly skilled professional musicians than in nonmusicians
(Bazanova et al., 2003), and IABW is positively correlated with the overall Torrance creativity coefficient and flexibility in creative task performance (Bazanova \& Aftanas, 2008), academic achievement (Bazanova \& Aftanas, 2006), and biofeedback training efficiency (Bazanova et al., 2007).

Research by D. A. Kaiser (2001) and Bazanova and Aftanas (2010) supported the importance of accounting for individual alpha band range in neurofeedback, showing that neurofeedback training applied in individual EEG frequency ranges was much more efficient than neurofeedback training of standard EEG frequency ranges (SNFT; Figure 4). The SNFT protocol of decreasing theta/beta ratio not only showed no psychometric improvement but also aggravated the clinical condition of the ADHD patient (Bazanova \& Aftanas, 2010). Moreover, we have shown that the enhancement of the individual alpha bandwidth is one of the main indicators of success in both neurofeedback and other modalities of biofeedback training (Bazanova et al., 2008; Bazanova et al.,
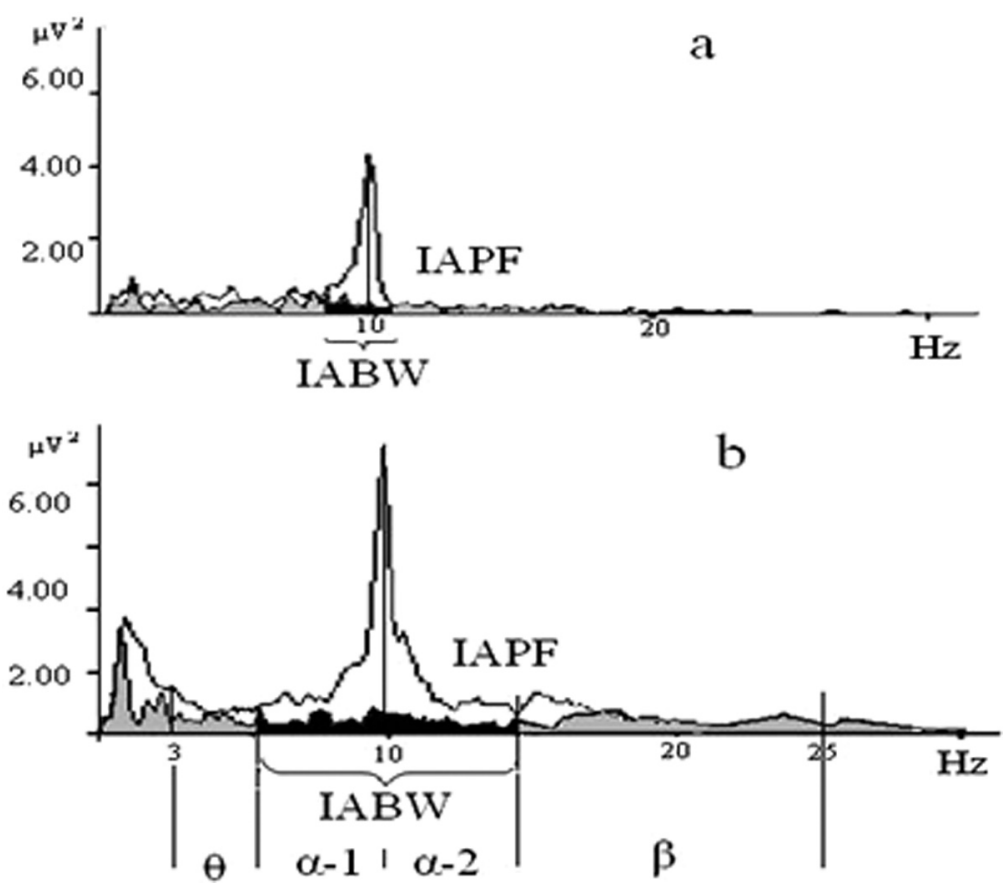

FIGURE 3. EEG spectral power in the parietal-occipital areas in the eyes-closed (white) and eyes-open (gray) conditions in healthy subjects. Note. Spectral power decreases by more than $20 \%$ from baseline within individual alpha ranges (black); (a) and (b) examples of different alpha-bandwidths. The abscissa shows frequency in $\mathrm{Hz}$; the ordinate shows spectral power at the frequencies indicated in $\mu \mathrm{V}^{2} ; \mathrm{IABW}=$ individual alpha bandwidth; IAPF = individual alpha peak frequency. 

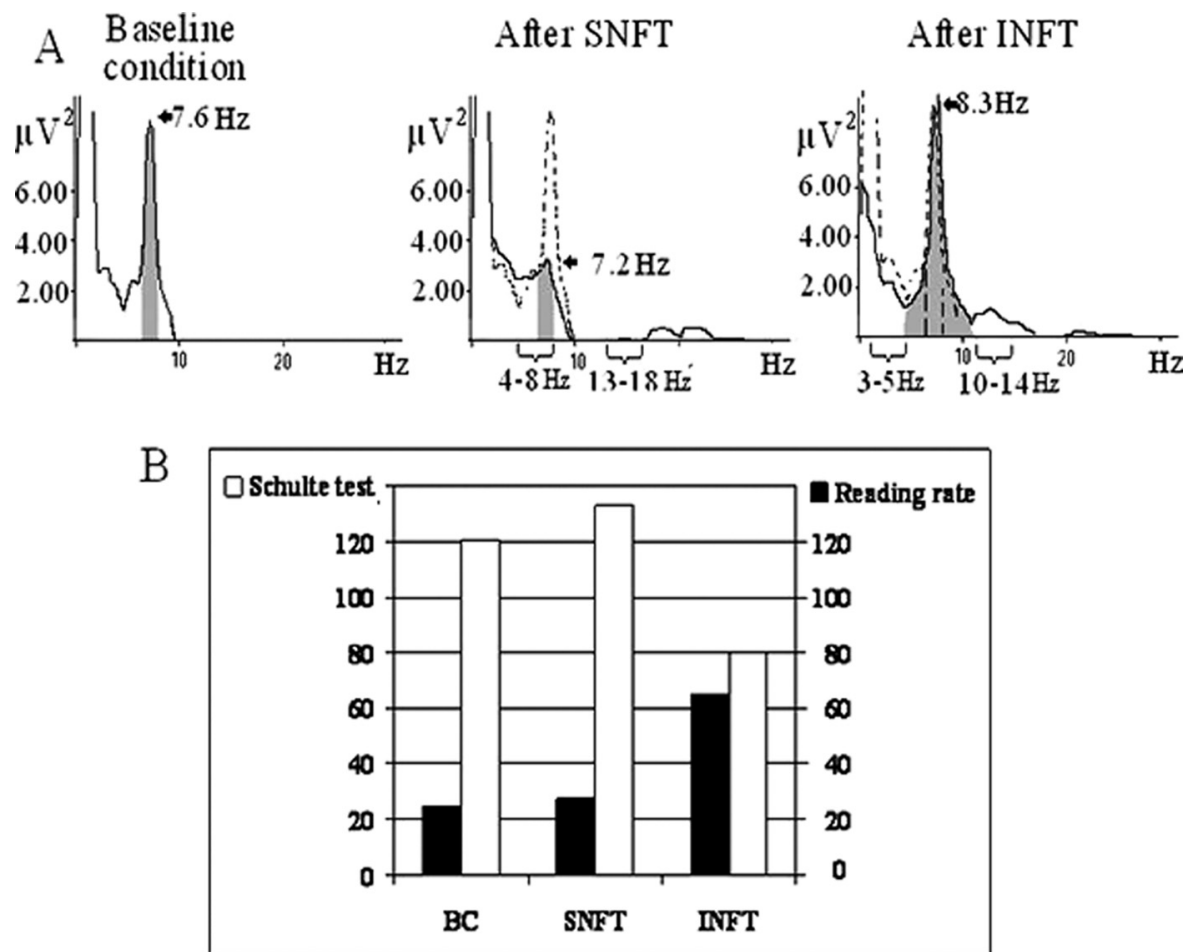

FIGURE 4. Spectral power (A) and psychometric test performance (B) of a boy with attention deficit disorder in baseline condition (BC), after 10 neurofeedback sessions decreasing the theta/beta ratio in standard EEG ranges (SNFT: theta $=4-8 \mathrm{~Hz}$ and beta $=13-18 \mathrm{~Hz}$ ) and in individual EEG ranges (INFT: theta $=3-5 \mathrm{~Hz}$, beta $=10-14 \mathrm{~Hz}$ ). Note. A: Gray areas denote spectral power in individual alpha band. Spectra power in baseline condition = dotted line; numbers with arrows=individual alpha peak frequency. B: Black bars indicate number of words read, and white bars indicate time to perform the task.

2007), indicating that the ability for enhancing self-control could be associated with the individual alpha band broadening (see Figure 4).

Activation magnitude measured as amount of alpha amplitude suppression and bandwidth during desynchronization raises many questions impossible to answer with simple spectral analysis. For example, it is not clear (a) whether the change of total power of particular brain alpha oscillations results from a change in the number of occurrences per minute rather than the change of the average oscillations amplitude, and (b) whether a change in the total power of alpha oscillations relates to the whole analyzed signal or rather to a small portion (Kaplan, Borisov, Shishkin, \& Ermolaev, 2002). Thus, regardless of how powerful or statistically significant the different estimations of averaged EEG effects may be, it is difficult to make meaningful interpretations if the estimations are not matched to the EEG piecewise stationary structure (Kaplan et al., 2002).
To overcome such limitations of conventional spectral analysis based on averaging procedures and to reveal dynamic and temporal characteristics of alpha activity, an entire set of individual short-term stationary EEG segments may be obtained (Brodsky et al., 1999; Towers \& Allen, 2009). Nonstationary phenomena are present in the EEG, usually in the form of transient events, such as alternation of relatively homogenous intervals (bursting segments) with different statistical features (e.g., with different amplitude or variance; Lopes da Silva, 1991). The idea that alpha oscillations have a spindle-like form only during sleep (Niedermeyer, 1999) has been contradicted by Kellaway (2003), who described the so-called lambda waves $(8-13 \mathrm{~Hz})$, which may be identified using simple procedures. The lambda wave is believed to represent occipital lobe activity in a person actively reading or scanning a room. Often, the subjects have their eyes open and are looking carefully 
at the ceiling tiles. The technician will have the patient reproduce the activities that they felt caused the waves to appear in the first place (Kellaway, 2003). It was proposed that the physiological basis of sleep spindles is probably very similar to lambda and alpha waves. To determine if the brain activation state would modulate the composition of alpha spatial microstates (spindles), Cantero and colleagues (1999) used spatial segmentation methods to show that (a) the mean duration of alpha spindles is longer in relaxed wakefulness than in drowsy periods and REM sleep, and (b) the number of different amplitude values are more abundant in drowsiness than in other brain states.

Thus, overall, brain activation mechanisms could become clearer if we consider the third important EEG alpha activity featurespindle-ability of alpha oscillation.

\section{AUTORHYTHMICITY}

The spindle-form or autorhythmicity of alpha oscillations is a product of the dynamics of neuronal assemblies in the underlying cortical activity (Dorokhov, 2003; Lehmann, Strik, Henggeller, \& Koukkou, 1994; Livanov \& Dumenko, 1987; W. Singer et al., 1997). Starting from Livanov's (1984) studies, it has been shown that spindle oscillations are essential for memory formation (Gais, Plihal, Wagner, \& Born, 2000; Lebedev, 1994, 2006), and associated with short- and mid-term synaptic plasticity (Steriade \& Timofeev, 2003). A probable molecular mechanism of these phenomena was proposed by Destexhe and Sejnowski (2003), who suggested that spindling may activate the protein kinase - a molecular "gate" - thus opening the door for gene expression and allowing long-term changes to take place following subsequent inputs.

Average amplitude within a segment indicates the volume of the neuronal population. The more neurons recruited into an assembly through local synchronization of their activity, the higher will be the oscillation amplitude of the corresponding assembly (Brodsky et al., 1999; Livanov \& Dumenko, 1987; Lopes da
Silva, 1991). The assumption that alpha amplitude reflects inhibition may, at first glance, appear contradictory to the idea that alpha plays an active role in information processing, but the idea is that inhibition is an important factor that controls the exact timing of an oscillation. Thus, inhibition helps to establish a highly selective activation pattern (Klimesch et al., 2007).

Average spindle duration represents the functional lifespan of the neuronal population or the duration of operations produced by such a population (Kaplan et al., 2002; Maltseva \& Masloboev, 1997). It has been shown that longer spindles indicate a more relaxed state (Huupponen et al., 2008). The spindle lifetime is correlated with fluency in cognitive task performance (Bazanova \& Aftanas, 2008), and efficiency of a single biofeedback training session (Bazanova et al., 2007; Bazanova et al., 2009). In addition, alpha-spindles are longer in highly skilled musicians than in amateurs (see Figure 5; Bazanova et al., 2009).

The shortest alpha segments belong to HAF subjects with the highest individual alpha peak frequencies and LAF subjects with the lowest individual alpha peak frequencies (Figure 5; Bazanova et al., 2008). The longest spindles belong to persons with an average, or approximately average, $10 \mathrm{~Hz}$ individual alpha peak

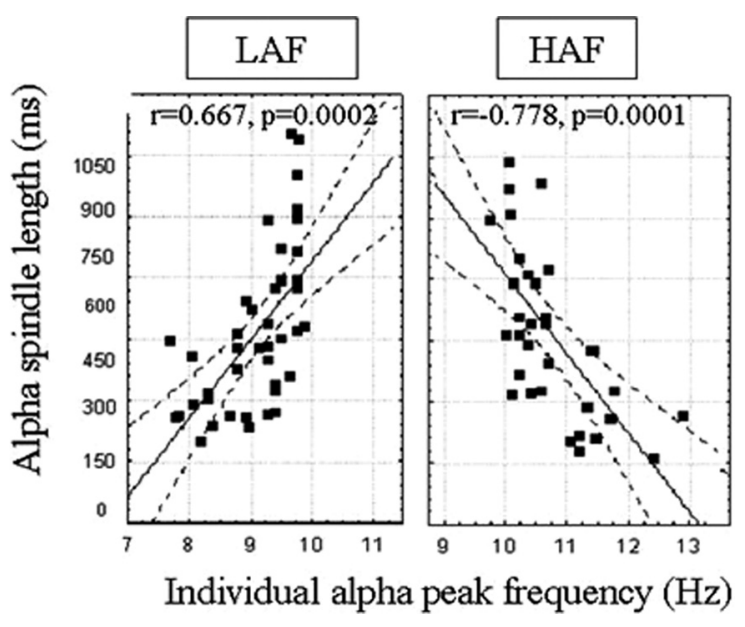

FIGURE 5. Results of regression analysis of the relationship between alpha spindle length and individual alpha peak frequency in low (LAF $n=48)$ and high (HAF $n=48)$ alpha frequency male subjects. 
frequency. Multiple regression analysis showed that spindle lifespan is positively related to individual alpha peak frequency in LAF subjects and negatively related in HAF subjects (Figure 5; Bazanova et al., 2008). These data suggest the different neuronal mechanism for spindle formation between LAF and HAF subjects. The membrane bistability might play an important role in different patterns of spindles displayed by thalamocortical neurons. Actually, as shown by Fuentealba and colleagues, the reticular neurons display membrane bistability, as indicated by two discrete electrical potential modes, with differential responsiveness to cortical inputs (Fuentealba, Timofeev, Bazhenov, Sejnowski, \& Steriade, 2005). In addition, in vivo (Steriade \& Llinas, 1988; Steriade \& Timofeev, 2003) and in vitro (Bal \& McCormick, 1996) intracellular studies have revealed at least two different patterns during spontaneously occurring spindles, which may be related to the actions exerted by nonbistable and bistable neurons, respectively. Indeed, nonbistable neurons fired stronger bursts with higher intraburst frequencies, which are assumed to generate IPSPs, $\sim 7-10 \mathrm{~Hz}$. By contrast, IPSPs with lower amplitudes and higher frequency are likely to be mainly generated by single action potentials, as they occur during the depolarizing plateau in bistable cells (Fuentealba et al., 2005). If we assume that longer spindles of stable brain activity imply less information to process (as reflected by a higher stability of the brain generator), whereas shorter segments imply a higher number of brain microstates caused by more different steps of information processing, then it is possible to suggest that the intrasegment alpha amplitude variability could be indexing a phasic event (Oprisan, Prinz, \& Canavier, 2004). Probably, alpha-bursts are associated with a brain microstate change (such as sleep spindles), as demonstrated by its phasic intrusion in a desynchronized background of brain activity (Cantero, Atienza, Salas, \& Gomez, 1999). It is important to know that intraspindle amplitude variability decreases in coma or stupor (Brenner, 2005) but increases during cognitive loading (Kaplan \& Borisov, 2003) and in relation to the ability for self-control in neurofeedback training (18-20 sessions; Bazanova et al., 2008). Therefore, amplitude variability, which is associated with phase resetting intensity (Oprisan et al., 2004), reflects engagement of cognitive control mechanisms (Hanlsmayr et al., 2007; Lebedev, 1994; Livanov \& Dumenko, 1987). Moreover, in children, the phase resetting index and intrasegment amplitude variability generally increases as a function of age (Bazanova, 2008; Thatcher et al., 2008). This probably reflects the well-known fact that ability for self-control develops with age (Mischel, 2004; Orekhova, Stroganova, Posikera, \& Malykh, 2003). Therefore, alpha activity indices, including individual alpha peak frequency, amount of alpha suppression and individual alpha bandwidth, and segmental parameters of alpha spindle characterize the degree of cortical ability for activation, neuronal plasticity, excitability, and inhibition. In this way these parameters could characterize self-regulation ability.

According to Klimesch's "time inhibition" theory, the active role of alpha waves is seen in a mechanism that may also underlie the functional role of other oscillations (Klimesch et al., 2007), whereas synchronization in the alpha frequency range helps neurons in distributed networks to effectively activate common target cells (Basar, 2006; Klimesch et al., 2007). This alpha-frequency dependent mechanism plays an important role in the top-down control of cortical activation. Mechanisms giving rise to alpha amplitude desynchronization also reflect the dependence of brain activation on the alpha frequency range. Klimesch has proposed that upper-frequency alpha oscillations are related to top-down processes in a complex sensory-motor system that controls the access to and manipulation of stored information (Klimesch et al., 2007). For example, when a task requires that certain types of processes be performed with stored information (e.g., stored information must be kept in mind, must not be retrieved or must be manipulated such as with highly skilled musicians during musical performance), individual alpha-activity increases. As with overall brain activity, 
top-down control is not a unitary phenomenon. Recently, Ben-Simon and associates (Ben-Simon, Podlipsky, Arieli, Zhdanov, \& Hendler, 2008) combined fMRI with EEG in a study that proposed two parallel patterns of alpha modulations and underpin their anatomical basis in the human brain. These findings suggest that the human alpha rhythm represents at least two simultaneously occurring processes that characterize the "resting brain"; one is related to expected change in sensory information, whereas the other is endogenous and independent of stimulus change. Although twin studies have long shown that heritability of EEG oscillations is substantial (Enoch et al., 2008; Smit et al., 2006), very little is known about the genes underlying distinct EEG traits. Early linkage analyses identified a genetic locus on the distal part of chromosome $20 q$ to modulate alpha power which authors named "alpha-activity" (Anokhin et al., 1992). It is likely, however, that multiple genes contribute to "alpha phenotypes," and a few candidate genes were indeed found to affect alpha oscillations. Recent genetic polymorphism studies indicated that the gene on chromosome 5q13-14 of corticotrophin releasing hormonebinding protein (CRH-BP) modulates alpha power in isolated Plains American Indians and Caucasians (Enoch et al., 2008). Moreover, a functional variation in exon 7 of the gene on chromosome 6 encoding the human GABA B receptor (GABABR1) also influences EEG voltage in the alpha range (Winterer et al., 2003). Finally, more recent data of Bodenmann et al. (2009) demonstrate that mechanisms involving COMT contribute to interindividual differences in alpha oscillation frequency, which are functionally related to executive performance (Bodenmann et al., 2009). Recent data by Aftanas et al. (2008) proposed that LAF and HAF subjects have different mechanisms for the modulatory influences of the balance of reinforcement system activities on autonomic vascular regulatory processes. Thus, the data just presented emphasize the genetically different EEG patterns and associated behavioral strategies dependent on alpha frequency.
In conclusion, studying the alpha EEG activity should not be limited only to alpha amplitude intra- and interindividual variability but should also include two other important physical characteristics: frequency and phase of alpha oscillations. Moreover, the evidence that alpha oscillations play an active role in cognitive processing and self-regulation is strongly related with the genotypic and phenotypic difference between low and high frequencies of the alpha activity. Moreover, the neuronal activation strategies for increasing IAPF as a result of biofeedback training are different according to the individual alpha frequency. Several factors may be common in the generation of different types of oscillations. Nonetheless, the exact mechanisms for generating an oscillation may differ widely between different frequency waves such as low- and high-frequency alpha, depending on individual network properties, cell types, cell physiology, hormone level, blood feeding, and so on. The role of such biological factors as immunological, neurohumoral, and activity of heart-vessel and breathing systems in the generation and formation alpha oscillations is still unknown. Thus, for a better understanding of the functional role of alpha activity indices in peak performance achievement through Biofeedback or $\mathrm{BCl}$ technologies, the investigation of cortico-visceral interplay in conjunction with the inherited alpha frequency EEG types may be of crucial importance.

\section{REFERENCES}

Aftanas, L. I., Sidorova, P. V., Pavlov, S. V., Makhnev, V. P., Korenek, V. V., Reva, N. V., \& Amstislavskaya, T. G. (2008). Activity of the positive and negative reinforcement motivation systems and baseline arterial blood pressure in humans. Neuroscience and Behavioral Physiology, 38, 799-806.

Angelakis, E., Lubar, J. F., \& Stathopoulou, S. (2004). Electroencephalographic peak alpha frequency correlates of cognitive traits. Neuroscience Letters, 371, 60-63.

Angelakis, E., Stathopoulou, S., \& Frymiare, J. L. (2007). EEG neurofeedback: A brief 
overview and an example of peak alpha frequency training for cognitive enhancement in the elderly. Clinical Neuropsychologist, 21, 110-129.

Anokhin, A. P., Muller, V., Lindenberger, U., Heath, A. C., \& Myers, E. (2006). Genetic influences on dynamic complexity of brain oscillations. Neuroscience Letters, 397, 93-98.

Anokhin, A., Steinlein, O., Fisher, C., Mao, Y., Vogt, P., Schalt, E., \& Vogel, F. A. (1992). Genetic study of the human low-voltage electroencephalogram. Human Genetics, 90, 99-112.

Arns, M., Gunkelman, J., Breteler, M., \& Spronk, D. (2008). EEG phenotypes predict treatment outcome to stimulants in children with ADHD. Journal of Integrative Neuroscience, 7, 421-438.

Baker, F. C., \& Colrain, I. M. (2010). Daytime sleepiness, psychomotor performance, waking EEG spectra and evoked potentials in women with severe premenstrual syndrome. Journal of Sleep Research, 19, 214-27.

Bal, T., \& McCormick, D. A. (1996). What stops synchronized thalamocortical oscillations? Neuron, 17, 297-308.

Barry, R. J., Clarke, A. R., \& Johnstone, S. J. (2003). A review of electrophysiology in attention-deficit/hyperactivity disorder: I. Qualitative and quantitative electroencephalography. Clinical Neurophysiology, 114, 171-183.

Barry, R. J., Clarke, A. R., Johnstone, S. J., Magee, C. A., \& Rushby, J. A. (2007). EEG differences between eyes-closed and eyesopen resting conditions. Clinical Neurophysiology, 118, 2765-2773.

Basar, E. (2006). The theory of the wholebrain-work. International Journal of Psychophysiology, 60, 133-138.

Baumeister, J., Reinecke, K., Liesen, H., \& Weiss, M. (2008). Cortical activity of skilled performance in a complex sports related motor task. European Journal of Applied Physiology, 104, 625-631.

Bazanova, O. M. (2008). Age related alpha activity change differs for males and females and for low and high alpha frequency EEG pattern. Revista Espanola de Neuropsicología, 10, 82-83.

Bazanova, O. M. (2011). Individual alpha peak frequency variability and reproducibility in various experimental conditions [Russian]. Zh Vyssh Nerv Deiat Im I P Pavlova, 61, 102-111.

Bazanova, O. M., \& Aftanas, L. I. (2006). Relationships between learnability and individual indices of EEG alpha activity. Annals of General Psychiatry, 5, 74-75.

Bazanova, O. M., \& Aftanas, L. I. (2008). Individual measures of electroencephalogram alpha activity and non-verbal creativity. Neuroscience and Behavioral Physiology, 38, 227-235.

Bazanova, O. M., \& Aftanas, L. I. (2010). Individual EEG alpha activity analysis for enhancement neurofeedback efficiency: Two case studies. Journal of Neurotherapy, 14, 244-253.

Bazanova, O. M., Grozdev, A. V., Mursin, F. A., Verevkin, E. G., \& Shtark, M. B. (2003). EEG-EMG dimensionality of the musical performance. Cognitive Processing, 4, 33-47.

Bazanova, O. M., Jafarova, O. A., Mazhirina, K. V., Mernaya, E. M., \& Shtark, M. B. (2008). Optimal functioning psychophysiological bases and neurofeedback training. International Journal of Psychophysiology, 69, 164-165.

Bazanova, O. M., Kondratenko, A., Kondratenko, O., Mernaya, E., \& Zhimulev, E. (2007). New computer-based technology to teach peak performance in musicians. Information Technology Interfaces, 7, 39-44.

Bazanova, O. M., \& Mernaya, E. M. (2008). Alpha-activity fluctuations in various hormonal states and associated with them musical performance proved differently in the opposite individual alpha peak frequency groups. Revista Espanola de Neuropsicología, 10, 100-101.

Bazanova, O. M., Mernaya, E. M., \& Shtark, M. B. (2009). Biofeedback in psychomotor training: Electrophysiological basis. Neuroscience and Behavioral Physiology, 39, 437-454.

Becker, D., Creutzfeldt, O. D., Schwibbe, M., \& Wuttke, W. (1980). Electrophysiological 
and psychological changes induced by steroid hormones in men and women. Acta Psychiatrica Belgica, 80, 674-697.

Ben-Simon, E., Podlipsky, I., Arieli, A., Zhdanov, A., \& Hendler, T. (2008). Never resting brain: Simultaneous representation of two alpha related processes in humans. PLOS ONE, 3, e3984. doi:10.1371/journal. pone.0003984

Bernstein, N. A. (1967). The co-ordination and regulation of movement. Oxford: Pergamon Press.

Bodenmann, S., Rusterholz, T., Dürr, R., Stoll, C., Bachmann, V., Geissler, E., ... Landolt, H. P. (2009). The functional Val158Met polymorphism of COMT predicts interindividual differences in brain alpha oscillations in young men. Journal of Neuroscience, 29, 10855-10862.

Brenner, R. P. (2005). The interpretation of the EEG in stupor and coma. Neurologist, 11, 271-284.

Brodsky, B. E., Darkhovsky, B. S., Kaplan, A. Y., \& Shishkin, S. L. (1999). A nonparametric method for the segmentation of the EEG. Comput Methods Programs Biomed, 60(2), 93-106.

Cantero, J. L., Atienza, M., Salas, R. M., \& Gomez, C. M. (1999). Brain spatial microstates of human spontaneous alpha activity in relaxed wakefulness, drowsiness period, and REM sleep. Brain Topography, 11, 257-263.

Carrier, J., Land, S., Buysse, D. J., Kupfer, D. J., \& Monk, T. H. (2001). The effects of age and gender on sleep EEG power spectral density in the middle years of life (ages 20-60 years old). Psychophysiology, 38, 232-242.

Clark, R. C., Veltmeyer, D., Hamilton, R. J., Simms, E., Paul, R., Hermens, D., \& Gordon, E. (2004). Spontaneous alpha peak frequency predicts working memory performance across the age span. International Journal of Psychophysiology, 53, 1-9.

Creutzfeldt, O. D., Arnold, P. M., Becker, D., Langenstein, S., Tirsch, W., Wilhelm, H., \& Wuttke, W. (1976). EEG changes during spontaneous and controlled menstrual cycles and their correlation with psychological performance. Electroencephalography and Clinical Neurophysiology, 40, 113-131.

Del Percio, C., Infarinato, F., Marzano, N., lacoboni, M., Aschieri, P., Lizio, R., ... Babiloni, C. (2011). Reactivity of alpha rhythms to eyes opening is lower in athletes than non-athletes: A high-resolution EEG study. International Journal of Psychophysiology, 82, 240-247.

Destexhe, A. \& Sejnowski, T. J. (2003). Interactions between membrane conductances underlying thalamocortical slow-wave oscillations. Physiological Reviews, 83, 1401-1453.

Doppelmayr, M., Klimesch, W., Hodlmoser, K., Sauseng, P., \& Gruber, W. (2005). Intelligence related upper alpha desynchronization in a semantic memory task. Brain Research Bulletin, 66, 171-177.

Dorokhov, V. B. (2003). Alpha-bursts and K-complex: Phasic activation pattern during spontaneous recovery of correct psychomotor performance at difference stages of drowsiness [Russian]. Zh Vyssh Nerv Deiat Im I P Pavlova, 53, 503-512.

Eccles, J. C. (1994). How the self controls its brain. Berlin, Germany: Springer-Verlag.

Enoch, M. A., Shen, P. H., Ducci, F., Yuan, Q., Liu, J., White, K. V., ... Goldman, D. (2008). Common genetic origins for EEG, alcoholism and anxiety: The role of CRH-BP. PLOS ONE, 3, e3620.

Fuentealba, P., Timofeev, I., Bazhenov, M., Sejnowski, T. J., \& Steriade, M. (2005). Membrane bistability in thalamic reticular neurons during spindle oscillations. Journal of Neurophysiology, 93, 294-304.

Gais, S., Plihal, W., Wagner, U., \& Born, J. (2000). Early sleep triggers memory for early visual discrimination skills. Nature Neuroscience, 3, 1335-1339.

Gavrish, N. V., \& Malykh, S. B. (1994). The nature of the variability in the individual differences of the frequency characteristics of the alpha-rhythm EEG in 6- to 8-year-old children [Russian]. Zh Vyssh Nerv Deiat Im I P Pavlova, 44, 8-17.

Hanslmayr, S., Klimesch, W., Sauseng, P., Gruber, W., Doppelmayr, M., Freunberger, R., ... Birbaumer, N. (2007). Alpha phase 
reset contributes to the generation of ERPs. Cerebral Cortex, 17, 1-8.

Hanslmayr, S., Sauseng, P., Doppelmayr, M., Schabus, M., \& Klimesch, W. (2005). Increasing individual upper alpha power by neurofeedback improves cognitive performance in human subjects. Applied Psychophysiology \& Biofeedback, 30, 1-10.

Hooper, G. S. (2005). Comparison of the distributions of classical and adaptively aligned EEG power spectra. Internal Journal of Psychophysiology, 55, 179-189.

Hummel, F., Saur, R., Lasogga, S., Plewnia, C., Erb, M., Wildgruber, D., \& Gerloff, C. (2004). To act or not to act: Neural correlates of executive control of learned motor behavior. Neurolmage, 23, 1391-1401.

Huupponen, E., Maksimow, A., Lapinlampi, P., Särkelä, M., Saastamoinen, A., Snapir, A. \& Jääskeläinen, S. (2008). Electroencephalogram spindle activity during dexmedetomidine sedation and physiological sleep. Acta Anaesthesiologica Scandinavia, 52, 289-294.

Ivanitsky, A. M., Ivanitsky, G. A., \& Sysoeva, O. V. (2009). Brain science: On the way to solving the problem of consciousness. International Journal of Psychophysiology, 73, 101-108.

Ivanitsky, A. M., \& Lebedev, A. N. (2007). Solving the riddle of the brain rhythms [Russian]. Zh Vyssh Nerv Deiat Im I P Pavlova, 57, 636-640.

Kaiser, D. A. (2001). Rethinking standard bands. Journal of Neurotherapy, 5, 96-101.

Kaiser, V., Clemens, B., Leeb, R., Neuper, C., \& Pfurtscheller, G. (2009). Investigation of cue-based vertical and horizontal eye movements with electroencephalographic and eye-tracking data. Clinical Neurophysiology, 120, 1988-1993.

Kaplan, A. Ia. (1999). The problem of the segmental description of the human electroencephalogram [Russian]. Fiziol Cheloveka, 25, 125-133.

Kaplan, A. la., \& Borisov, S. V. (2003). Dynamic properties of segmental characteristics of EEG alpha activity in rest conditions and during cognitive tasks [Russian]. Zh Vyssh Nerv Deiat Im I P Pavlova, 53, 22-32.
Kaplan, A. la., Borisov, S. V., Shishkin, S. L., \& Ermolaev, V. A. (2002). Analysis of the segmental structure of EEG alpha-activity in humans [Russian]. Ross Fiziol Zh Im I M Sechenova, 88, 432-442.

Keefe, T. (1978). Optimal functioning: The Eastern ideal in psychotherapy. Journal of Contemporary Psychotherapy, 10, 16-24.

Kellaway, P. (2003). Orderly approach to visual analysis: Elements of the normal EEG and their characteristics in children and adults. In J. S. Ebersole \& T. A. Pedley (Eds.), Current practice of clinical electroencephalography (3rd ed., pp. 100-159). Philadelphia, PA: Lippincott Williams and Wilkins.

Kirschfeld, K. (2005). The physical basis of alpha waves in the electroencephalogram and the origin of the "Berger effect." Biological Cybernetics, 92, 177-185.

Klimesch, W., Sauseng, P., \& Hanslmayr, S. (2007). EEG alpha oscillations: The inhibition-timing hypothesis. Brain Research Reviews, 53, 63-88.

Klimesch, W., Schimke, H., \& Pfurtscheller, G. (1993). Alpha frequency, cognitive load and memory performance. Brain Topography, 5, 241-251.

Laufs, H., Holt, J. L., Eltont, R., Krams, M., Paul, J. S., Krakow, K., \& Kleinschmidt, A. (2006). Where the BOLD signal goes when alpha EEG leaves. Neuroimage, 31, 1408-1418.

Lebedev, A. N. (1994). The neurophysiological parameters of human memory. Neuroscience and Behavioral Physiology, 24, 254-259.

Lebedev, A. N. (2006). Mikhail Nikolaevich Livanov (on his 100th anniversary of his scientific, scientific-organizational, pedagogical and public activities) [Russian]. Usp Fiziol Nauk, 37, 87-94.

Lehmann, D., Strik, W. K., Henggeller, B., \& Koukkou, M. (1994). Microstates in spontaneous momentary EEG potential maps during visual imagery and abstract thought. Brain Topography, 6, 251.

Livanov, M. N. (1984). Rhythms of the electroencephalogram and their functional significance [Russian]. Zh Vyssh Nerv Deiat Im I P Pavlova, 34, 613-626. 
Livanov, M. N., \& Dumenko, V. N. (1987). The neurophysiological aspect of research on the systems organization of brain activities [Russian]. Usp Fiziol Nauk, 18, 6-16.

Loo, S. K., \& Smalley, S. L. (2008). Preliminary report of familial clustering of EEG measures in ADHD. American Journal of Medical Genetics Part B: Neuropsychiatric Genetics, 147B, 107-109.

Loo, S. K., Hale, S. T., Hanada, G., Macion, J., Shrestha, A., McGough, J. J., \& Smalley, S. L. (2010). Familial clustering and DRD4 effects on electroencephalogram measures in multiplex families with attention deficit/hyperactivity disorder. Journal of the American Academy of Child and Adolescent Psychiatry, 49, 368-377.

Lopes da Silva, F. H. (1991). Neural mechanisms underlying brain waves: From neural membranes to networks. Electroencephalography and Clinical Neurophysiology, 79, 81-93.

Lüthi, A., \& McCormick, D. A. (1998). $\mathrm{H}$-current: Properties of a neuronal and network pacemaker. Neuron, 21, 9-12.

Maltseva, I. V., \& Masloboev, Y. P. (1997). Alpha rhythm parameters and short-term memory span. International of Journal Psychophysiology, 26, 369-380.

Mantanus, H., Ansseau, M., Legros, J. J., \& Timsit-Berthier, M. (1988). Relationship between dexamethasone suppression test and contingent negative variation in major depressive patients [French]. Neurophysiologie Clinique, 18, 345-353.

Mazaheri, A., \& Jensen, O. (2006). Posterior alpha activity is not phase-reset by visual stimuli. Proceedings of the National Academy of Sciences, 103, 2948-2952.

Mischel, W. (2004). Toward an integrative science of the person. Annual Review of Psychology, 55, 1-22.

$\mathrm{Ng}$, S. C., \& Raveendran, P. (2007). EEG peak alpha frequency as an indicator for physical fatigue. International Federation for Medical and Biological Engineering Proceedings, 16, 517-520.

Niedermeyer, E. (1999). The normal EEG of the waking adult. In E. Niedermeyer \& F. Lopes da Silva (Eds.), Electroencephalography: Basic principles, clinical applications and related fields (4th ed., pp. 149-173). Philadelphia, PA: Williams and Wilkins.

Nunez, P., Wingeier, B., \& Silberstein, R. (2001). Spatial-temporal structures of human alpha rhythms: Theory, microcurrent sources, multiscale measurements, and global binding of networks. Human Brain Mapping, 13, 125-164.

Oprisan, S. A., Prinz, A. A., \& Canavier, C. C. (2004). Phase resetting and phase locking in hybrid circuits of one model and one biological neuron. Biophysical Journal, 87, 2283-2298.

Orekhova, E. V., Stroganova, T. A., Posikera, I. N., \& Malykh, S. B. (2003). Heritability and "environmentability" of electroencephalogram in infants: The twin study. Psychophysiology, 40, 727-741.

Pfurtscheller, G., \& Lopes da Silva, T. H. (1999). Event-related EEG/MEG synchronization and desynchronization: Basic principles. Clinical Neurophysiology, 110, 1842-1857.

Posthuma, D., Neale, M. C., Boomsma, D. I., \& de Geus, E. J. C. (2001). Are smarter brains running faster? Heritability of alpha peak frequency, IQ, and their interrelation. Behavior Genetics, 31, 567-587.

Shmelkina, R. (1999). Some EEG findings caused by real and imaginary stimuli in patients and healthy subjects. Applied Psychophysiology and Biofeedback, 24(2), 143-149.

Singer, R. N. (2002). Preperformance state, routines, and automaticity: What does it take to realize expertise in self-paced events? Journal of Sport \& Exercise Psychology, 24, 359-375.

Singer, W., Engel, A. K., Kreiter, A. S., Munk, M. H., Neuenschwander, S., \& Roelfsema, P. R. (1997). Neuronal assemblies: Necessity, signature and detectability. Trends in Cognitive Science, 1, 252-261.

Smit, C. M., Wright, M. J., Hansell, N. K., Geffen, G. M., \& Martin, N. G. (2006). Genetic variation of individual alpha frequency (IAF) and alpha power in a large adolescent twin sample. International Journal of Psychophysiology, 61, 235-243. 
Solis-Ortiz, S., Guevara, M. A., \& CorsiCabrera, M. (2004). Performance in a test demanding prefrontal functions is favored by early luteal phase progesterone: An electroencephalographic study. Psychoneuroendocrinology, 29, 1047-57.

Steriade, M. (1999). Brainstem activation of thalamocortical systems. Brain Research Bulletin, 50(5-6), 391-392.

Steriade, M., \& Llinas, R. R. (1988). The functional states of the thalamus and the associated neuronal interplay. Physiological Reviews, 68, 649-742.

Steriade, M., \& Timofeev, I. (2003). Neuronal plasticity in thalamocortical networks during sleep and waking oscillations. Neuron, 37, 563-76.

Stroganova, T. A., Orekhova, E. V., \& Posikera, I. N. (1999). EEG alpha rhythm in infants. Clinical Neurophysiology, 110, 997-1012.

Tenke, C. E., \& Kayser, J. (2005). Reference-free quantification of EEG spectra: Combining current source density (CSD) and frequency principal components analysis (fPCA). Clinical Neurophysiology, 116, 2826-2846.

Thatcher, R. W., North, D. M., \& Biver, C. J. (2008). Intelligence and EEG phase reset: A two compartmental model of phase shift and lock. Neurolmage, 42, 1639-1653.

Thuraisingham, R. A., Tran, Y., Boord, P., \& Craig, A. (2007). Analysis of eyes open, eye closed EEG signals using second-order difference plot. Medical \& Biological Engineering \& Computing, 45, 1243-1249.

Timofeev, I., Grenier, F., Bazhenov, M., Houweling, A. R., Sejnowski, T. J., \& Steriade, M. (2002). Short- and mediumterm plasticity associated with augmenting responses in cortical slabs and spindles in intact cortex of cats in vivo. Journal of Physiology, 542(Pt. 2), 583-598.

Tops, M., van Peer, J. M., Wester, A. E., Wijers, A. A., \& Korf, J. (2006). State-dependent regulation of cortical activity by cortisol: An EEG study. Neuroscience Letters, 404, 39-43.

Toscani, M., Marzi, T., Righi, S., Viggiano, M. P., \& Baldassi, S. (2010). Alpha waves: A neural signature of visual suppression. Experimental Brain Research, 207, 213-219.

Towers, D. N., \& Allen, J. J. (2009). A better estimate of the internal consistency reliability of frontal EEG asymmetry scores. Psychophysiology, 46, 132-142.

Vaez Mousavi, S. M., Barry, R. J., Rushby, J. A., \& Clarke, A. R. (2007). Arousal and activation effects on physiological and behavioral responding during a continuous performance task. Acta Neurobiologiae Experimentalis, 67, 461-470.

Vernon, D. (2009). Human potential: Exploring techniques used to enhance human performance. London, UK: Routledge.

Vernon, D., \& Gruzelier, J. (2008) Electroencephalographic biofeedback as a mechanism to alter mood, creativity and artistic performance. In B. N. DeLuca (Ed.), Mind-body and relaxation research focus (pp. 149164). Hauppauge, NY: Nova Science.

Winterer, G., Mahlberg, R., Smolka, M. N., Samochowiec, J., Ziller, M., Rommelspacher, H. P., ... Sander, T. (2003). Association analysis of exonic variants of the GABA(B)receptor gene and alpha electroencephalogram voltage in normal subjects and alcoholdependent patients. Behavioral Genetics, 33, 7-15. 